\title{
ON GENERALIZED PEANO DERIVATIVES
}

BY

CHENG-MING LEE

\begin{abstract}
A function $F$ is said to have a generalized $n$th Peano derivative at $x$ if $F$ is continuous in a neighborhood of $x$ and if there exists a positive integer $k$ such that a $k$ th primitive of $F$ in the neighborhood has the $(k+n)$ th Peano derivative at $x$; and in this case this $(k+n)$ th Peano derivative at $x$ is proved to be independent of the integer $k$ and the $k$ th primitives, and is called the generalized $n$th Peano derivative of $F$ at $x$ which is denoted as $F_{[n]}(x)$. If $F_{[n]}(x)$ exists and is finite for all $x$ in an interval, then it is shown that $F_{[n]}$ shares many interesting properties that are known for the ordinary Peano derivatives. Using the generalized Peano derivatives, a notion called absolute generalized Peano derivative is studied. It is proved that on a compact interval, the absolute generalized Peano derivatives are just the generalized Peano derivatives. In particular, Laczkovich's absolute (ordinary) Peano derivatives are generalized Peano derivatives.
\end{abstract}

1. Introduction. In obtaining an integral which is more general than any integral in the scale of the Cesaro-Perron integrals due to Burkill [5], a notion called the generalized Peano derivative has been introduced in [9]. The purpose of this note is to give a rather detailed and self-contained investigation to some of the elementary and basic properties for this generalized Peano derivative which were not touched or only briefly mentioned in [9]. Hopefully, it will provide a good ground for further studies of such generalized derivatives and their related integration theories.

A function $F$ is said to have a generalized $n$th Peano derivative at $x$ if $F$ is continuous on a neighborhood of $x$ and if there exists a positive integer $k$ such that a $k$ th primitive of $F$ on the neighborhood of $x$ has the ordinary $(k+n)$ th Peano derivative at $x$; and in this case, the $(k+n)$ th Peano derivative of the $k$ th primitive at $x$ is called the generalized $n$th Peano derivative at $x$, and is denoted as $F_{[n]}(x)$. As mentioned in [9], the number $F_{[n]}(x)$, if it exists, is indepedent of the integer $k$ and the $k$ th primitives. This fact follows from a basic result for ordinary Peano derivatives which is proved as Lemma 1 in $\$ 2$, where some other results on the ordinary Peano derivative which are relevant to our development here are also reviewed.

Generalizing some results known for the ordinary Peano derivatives, we prove that if $F_{[n]}(x)$ exists and is finite for all $x$ in an interval, then on this interval one has

(I) $F_{[n]}$ is in the first class of Baire;

(II) $F_{[n]}$ has the Darboux property;

Received by the editors November 17, 1981 and, in revised form, January 25, 1982

1980 Mathematics Subject Classification. Primary 26A24; Secondary 26A39, 26A48.

Key words and phrases. Peano derivative, generalized Peano derivative, absolute Peano derivative, absolute generalized Peano derivative, Cesaro-Perron integrals, Darboux property, Denjoy property, approximate Peano derivative. 
(III) $F_{[n]}$ has the Denjoy property;

(IV) if $F_{[n]}$ is bounded on one side, then $F_{[n]}$ is just the ordinary $n$th derivative of F.

The properties (I) and (II) have been proved in [9]. The proof of (I) is, however, reproduced here (Theorem 1) for the sake of completeness. The proof of (II) in [9] is somehow indirect since it was done there by using Lojasiewicz's work [14] heavily related to Schwartz's distribution theory. Here, a new and direct proof is given. In fact, both (II) and (III) (see Theorem 4) follow in a standard manner from (I) and (IV). The property (IV) is proved in conjunction with a monotonicity theorem for the generalized Peano derivative (see Theorem 3), which extends a very general result by Verblunsky in [21] for the ordinary Peano derivative.

The results mentioned above are genuine generalizations of those known for the ordinary Peano derivatives since there are generalized Peano derivatives on an interval which are not exact ordinary Peano derivatives there (see Remark 4). Even more is true. In fact, generalizing the concept of the absolute Peano derivative by Laczkovich [8], we study the absolute generalized Peano derivative (see Definition 2) in $\S 4$. It is shown that the absolute generalized Peano derivatives on a compact interval are just the generalized Peano derivatives there (see the corollary to Theorem 7). In particular, a Laczkovich's absolute Peano derivative on a compact interval is a generalized Peano derivative there, and hence has all the properties (I)-(IV) listed in the last paragraph. It should be remarked that the development of the absolute generalized Peano derivatives in $\$ 4$ follows the same line as that in [8]. On the other hand, the methods (see §3) used in establishing the properties (I)-(IV) and others for the generalized Peano derivatives (and hence, in particular, for the absolute Peano derivatives) are quite different from those used by Laczkovich in [8] for his absolute Peano derivatives.

The ordinary Peano derivative is known to have many other interesting and deeper properties such as the Zahorski property, the property $Z$ (see Weil $[23,24]$, and also Babcock [1] for extensions to the approximate Peano derivatives), and the oscillatory behavior obtained by O'Malley and Weil in [17] (see also [12]). Whether the generalized Peano derivative has the same properties still remains to be seen. Also, whether it is possible to obtain a "genuine (absolute) generalized" approximate Peano derivative is still an open question. These two respects are somehow discussed in the last section.

2. Peano derivatives. Let $F$ be a real-valued function defined on a neighborhood of the point $x$. The upper 1st Peano derivate of $F$ at $x$, denoted as $u F_{(1)}(x)$, is just the ordinary upper derivate of $F$ at $x$, i.e.

$$
u F_{(1)}(x)=\lim \sup [F(x+h)-F(x)] / h
$$

where the lim sup is taken as $h \rightarrow 0$. The lower 1st Peano derivate at $x$, denoted as $l F_{(1)}(x)$, is defined to be the liminf of the same difference quotient. If $u F_{(1)}(x)=$ $l F_{(1)}(x)$, the common value, denoted as $F_{(1)}(x)$, is called the 1st Peano derivative of $F$ at $x$, which is just the ordinary derivative of $F$ at $x$ and hence will also be denoted as $F^{\prime}(x)$ or $F^{(1)}(x)$. If $F_{(1)}(x)$ exists and is finite, the function $F$ is said to be 1 st Peano 
differentiable at $x$ (or simply that $F$ is differentiable at $x$ ). [Note that if $F$ is differentiable at $x$, then it is continuous at $x$. However, if $F_{(1)}(x)$ exists and is infinite, the function $F$ may not be continuous at $x$.] Inductively, let $k>1$, and suppose that $f_{(i)}(x)$ exists and is finite for $1 \leqslant i<k$. Then the upper $k$ th Peano derivate of $F$ at $x$, denoted as $u F_{(k)}(x)$, is defined as the limsup (as $h \rightarrow 0$ ) of the following expression:

$$
\left[F(x+h)-F(x)-\sum_{i=1}^{k-1}\left(h^{i} / i !\right) F_{(i)}(x)\right] k ! / h^{k} .
$$

Similarly, $l F_{(k)}(x)$ is defined by taking the liminf of the same expression. If $u F_{(k)}(x)=l F_{(k)}(x)$, the common value is called the $k$ th Peano derivative of $F$ at $x$ and is simply denoted as $F_{(k)}(x)$. If $F_{(k)}(x)$ exists and is finite, the function $F$ is said to be $k$ th Peano differentiable at $x$. [Note that if $F_{(k)}(x)$ exists and $k>1$, then $F_{(1)}(x)$ exists and is finite so that $F$ is continuous at $x$ even if $F_{(k)}(x)$ is infinite.]

It is often more convenient to denote $F(x)$ as $F_{(0)}(x)$ or $F^{(0)}(x)$ when $F$ is continuous at $x$, and in this case we may say that $F$ is 0 th differentiable at $x$ with $F_{(0)}(x)=\lim _{h \rightarrow 0} F(x+h)=F(x)$ as the 0th (Peano) derivative of $F$ at $x$.

The one-sided notion is obtained by suitably restricting the sign of $h$ in the above definitions. Thus, the meaning of $u F_{(k),+}(x), F_{(k),+}(x)$, etc. should be clear when we use them later. The repeated $k$ th ordinary derivative of $F$ at $x$ is to be denoted as $F^{(k)}(x)$. It is clear that if $F^{(k)}(x)$ exists, then $F_{(k)}(x)$ exists and equals $F^{(k)}(x)$. The converse is true only when $k=1$. (For a partial converse, see Theorem $\mathrm{B}_{n}$ below.)

For many interesting properties on Peano derivatives, we refer to $[2-6,16,17,19$ 24]. Here we recall the following results, which are essentially what we want to extend further later on:

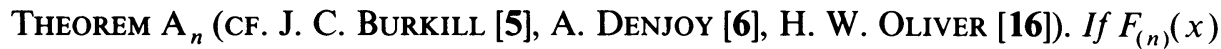
exists and is finite for all $x$ in an interval, then $F_{(n)}$ is in the first class of Baire on the interval.

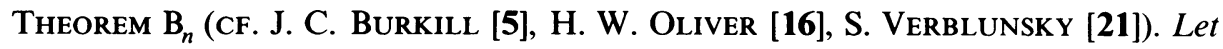
$F$ be nth Peano differentiable on an interval. If $F_{(n)}$ is bounded on one side on the interval, then $F^{(n)}$ exists and equals $F_{(n)}$ on the interval.

Theorem $\mathrm{C}_{n}$ (CF. S. Verblunsky [21], AND Also [10 OR 15]). Let $F$ be nth Peano differentiable on $[a, b]$. If $u F_{(n+1)}(x) \geqslant 0$ for almost all $x$ in $[a, b]$ and $u F_{(n+1)}(x)>-\infty$ for nearly all $x$ (i.e. for all except possibly on a countable set of points) in $[a, b]$, then $F_{(n)}$ is monotone nondecreasing and continuous on $[a, b]$.

Remark 1. In the above definitions of Peano derivates if the ordinary lim sup and lim inf are replaced by the approximate ones (i.e. lim sup ap and lim inf ap as given in Saks' book [18]), one has the definitions for the approximate Peano derivates. The 1st approximate Peano derivative has been classical, while the higher order ones have been observed recently by many people. Theorems $\mathrm{A}_{n}, \mathrm{~B}_{n}$ and $\mathrm{C}_{n}$, as well as many others concerning Peano derivatives, have been generalized for the approximate Peano derivatives $($ see $[7,1,15,10,12])$. We will show that many of the results 
can be generalized for the generalized Peano derivatives considered in [9]. The development is based on the following result, which is a consequence of the Bernoulli-l'Hospital rule. However, for the sake of completeness, we give a selfcontained proof here. It is interesting to know that the result fails to hold true if the ordinary Peano derivatives are replaced by the approximate ones (e.g. see [13 or 8]).

LEMMA 1. Let $f$ and $g$ be real-valued functions such that $g_{(1)}=f$ on a neighborhood of $x$. Suppose that $u f_{(n)}(x)$ makes sense for a positive integer $n$. Then $u g_{(n+1)}(x)$ makes sense and

$$
l f_{(n)}(x) \leqslant \lg _{(n+1)}(x) \leqslant u g_{(n+1)}(x) \leqslant u f_{(n)}(x) .
$$

In particular, if $f_{(n)}(x)$ exists, then $g_{(n+1)}(x)$ exists and equals $f_{(n)}(x)$.

Proof. The proof is by induction on $n$. Since $u g_{(2)}$ makes sense trivially, for $n=1$, it suffices to show that

$$
l f_{(1)}(x) \leqslant l g_{(2)}(x) \leqslant u g_{(2)}(x) \leqslant u f_{(1)}(x) .
$$

We will only prove the inequality $u g_{(2)}(x) \leqslant u f_{(1)}(x)$ since then the inequality $l f_{(1)}(x) \leqslant \lg _{(2)}(x)$ will follow immediately by considering the functions $-f$ and $-g$. To show that $u g_{(2)}(x) \leqslant u f_{(1)}(x)$, we may assume that $u f_{(1)}(x)$ is not $+\infty$ for, otherwise, the inequality holds automatically. Let $\alpha$ be any arbitrary real number such that $u f_{(1)}(x)<\alpha$. Then there exists a neighborhood $I$ of 0 such that

$$
[f(x+h)-f(x)] / h<\alpha
$$

for all $h \in I$ with $h \neq 0$. On $I$, consider the function $G$ defined by

$$
G(h)=g(x+h)-g(x)-h f(x)-\alpha h^{2} / 2
$$

for each $h \in I$. As $G^{\prime}(h)=f(x+h)-f(x)-\alpha h$, one sees from (1) that $G$ is decreasing on $I \cap[0,+\infty]$ and increasing on $I \cap(-\infty, 0]$. Therefore, $G(h) \leqslant G(0)$ $=0$ for all $h \in I$. Then

$$
[g(x+h)-g(x)-h f(x)] 2 / h^{2} \leqslant \alpha
$$

for all $h \in I$ with $h \neq 0$. Hence $u g_{(2)}(x) \leqslant \alpha$. Since $\alpha$ is an arbitrary real number greater than $u f_{(1)}(x)$, one concludes that $u g_{(2)}(x) \leqslant u f_{(1)}(x)$, completing the proof for the case $n=1$.

Now let $k$ be any integer greater than 1, and assume that the lemma holds for $n<k$. We want to show that the lemma holds for $n=k$. Since $u f_{(k)}(x)$ makes sense, $f_{(n)}(x)$ exists and is finite for $n<k$. Then, by the induction hypothesis, $g_{(n+1)}(x)$ exists and equals $f_{(n)}(x)$ for $n<k$, and hence $u g_{(k+1)}(x)$ makes sense. It remains to show that the inequalities stated in the lemma hold for $n=k$. As before, it suffices to show that $u g_{(k+1)}(x) \leqslant u f_{(k)}(x)$ under the assumption that $u f_{(k)}(x)<+\infty$. Let $\alpha$ be an arbitrary real number greater than $u f_{(k)}(x)$. Then there exists a neighborhood $I$ of 0 such that

$$
\left[f(x+h)-f(x)-\sum_{i=1}^{k-1} f_{(i)}(x) h^{i} / i !\right] k ! / h^{k}<\alpha
$$


for all $h \in I$ with $h \neq 0$. Then consider the function $G$ defined by

$$
G(h)=g(x+h)-g(x)-\sum_{i=1}^{k} g_{(i)}(x) h^{i} / i !-\alpha h^{k+1} /(k+1) !
$$

for $h \in I$. Note that

$$
G^{\prime}(h)=f(x+h)-f(x)-\sum_{i=1}^{k-1} f_{(i)}(x) h^{i} / i !-\alpha h^{k} / k !,
$$

so that it follows from (2) that $G$ is decreasing on $I$ when $k$ is even, and $G$ is decreasing on $I \cap[0,+\infty)$ and increases on $I \cap(-\infty, 0]$ when $k$ is odd. Then one concludes that, either $k$ is even or odd,

$$
\left[g(x+h)-g(x)-\sum_{i=1}^{k} g_{(i)}(x) h^{i} / i !\right](k+1) ! / h^{k+1}<\alpha,
$$

so that $u g_{(k+1)}(x) \leqslant \alpha$. Hence $u g_{(k+1)}(x) \leqslant u f_{(k)}(x)$. This completes the proof.

REMARK 2. The converse of Lemma 1 is not true since there are functions $f$ and $g$ such that $g_{(1)}=f$ on a neighborhood of $x$ and $g_{(n+1)}(x)$ exists while $f_{(n)}(x)$ does not exist. For example, let $g(x)=x^{3} \sin (1 / x)$ and $f(x)=3 x^{2} \sin (1 / x)-x \cos (1 / x)$ when $x \neq 0$, and let $f(0)=g(0)=0$; then $g_{(1)}=f$ and $g_{(2)}(0)=0$ but $f_{(1)}(0)$ does not exist. More generally, the $n$th Peano derivative of a function may not exist while its $k$ th primitive may have the $(k+n)$ th Peano derivative at the particular point.

3. Generalized Peano derivatives. For convenience, if $F$ is a continuous function on an interval $I$, we will write $F^{(-0)}=F^{(0)}=F$ on $I$, and for any integer $k \geqslant 1$, we denote

$$
F^{(-k)}(x)=\int^{x} F^{(-k+1)}(t) d t
$$

for all $x \in I$, i.e. $F^{(-k)}$ is an indefinite Riemann integral of the function $F^{(-k+1)}$ on $I$. Thus, $F^{(-k)}$ is just a $k$-fold indefinite Riemann integral of the continuous function $F$ on the interval concerned. Note that two $k$-fold indefinite integrals of the same function on an interval can be different by at most a term which is a polynomial of degree less than $k$. Furthermore, if $G=F^{(-k)}$, then $G^{(i)}=F^{(-k+i)}$ for $0 \leqslant i \leqslant k$, and $G_{(k+1)}(x)$, if it exists, is independent of which one of the $k$-fold indefinite Riemann integrals of $F$ is taken as $F^{(-k)}$.

Throughout the rest of this section, $n$ will stand for a fixed but arbitrary positive integer unless it is otherwise specified.

Definition 1. A real-valued function $F$ is said to have a generalized $n$th Peano derivative at $x$ if there exists a nonnegative integer $k$ such that the function $F^{(-k)}$ on a neighborhood of $x$ has the $(k+n)$ th Peano derivative at $x$, and this $(k+n)$ th Peano derivative of $F^{(-k)}$ at $x$ is called a generalized $n$th Peano derivative of $F$ at $x$.

Note that if the $(k+n)$ th Peano derivative of an $F^{(-k)}$ exists at $x$ and if $l$ is an integer not less than $k$, then it follows from Lemma 1 that the $(l+k)$ th Peano derivative at $x$ of any $l$-fold indefinite Riemann integral of $F$ exists and equals $F_{(k+n)}^{(-k)}(x)$. Hence, if a function $F$ has $a$ generalized $n$th Peano derivative at $x$, it has a unique one, and this unique generalized $n$th Peano derivative of $F$ at $x$ is denoted as 
$F_{[n]}(x)$. If $F_{[n]}(x)$ exists and is finite, we say that $F$ is generalized nth Peano differentiable at $x$ (cf. [9], where the definition of the finite generalized $n$th Peano derivative was given). For convenience, when a function $F$ is continuous at $x$, we will also say that $F$ is generalized 0th (Peano) differentiable at $x$, and its 0 th derivative there is just $\lim _{h \rightarrow 0} F(x+h)(=F(x))$, which will sometimes also be denoted as $F_{[0]}(x)$ or $F_{(0)}(x)$ or $F^{(0)}(x)$.

Note that for a function $F$ which is continuous in a neighborhood of $x$, if $F_{(n)}(x)$ exists, then $F_{[n]}(x)$ exists and equals $F_{(n)}(x)$. But the converse is not true as the example in Remark 2 shows. Also note that it follows from Definition 1 that if $F_{[n]}(x)$ exists then for any positive integer $k,\left(F^{(-k)}\right)_{[n+k]}(x)$ exists and equals $F_{[n]}(x)$; but $\left(F^{(-k)}\right)_{(n+k)}$ may not exist unless $k$ is large enough (see Lemma 2 below).

Note also that if $F_{[n]}(x)$ exists, then the function $F$ is generalized $(n-1)$ th Peano differentiable at $x$. On the other hand, if $F$ is generalized $(n-1)$ th differentiable at $x$, let us introduce the following notations:

$$
u_{0} F_{[n]}(x)=\lim \sup \left[F(x+h)-\sum_{i=0}^{n-1} F_{[i]}(x) h^{i} / i !\right] n ! / h^{n},
$$

and $l_{0} F_{[n]}(x)$ is the lim inf of the same quotient, where the limit processes are taken as $h \rightarrow 0$. The following simple results are helpful in seeing what are involved in Definition 1.

Lemma 2. Let $F$ be generalized nth Peano differentiable at $x$. Then each of the following holds true:

(i) If both $l_{0} F_{[n+1]}(x)$ and $u_{0} F_{[n+1]}(x)$ are finite, then $F$ is (ordinarily) nth Peano differentiable at $x$.

(ii) There exists a nonnegative integer $k_{0}$ such that whenever $k \geqslant k_{0}$, $l\left(F^{(-k)}\right)_{(k+n+1)}(x)$ makes sense and

(3) $l_{0} F_{[n+1]}(x) \leqslant l\left(F^{(-k)}\right)_{(k+n+1)}(x) \leqslant u\left(F^{(-k)}\right)_{(k+n+1)}(x) \leqslant u_{0} F_{[n+1]}(x)$.

In particular, if $l_{0} F_{[n+1]}(x)=u_{0} F_{[n+1]}(x)$, then $F_{[n+1]}(x)$ exists and equals $l_{0} F_{[n+1]}(x)$.

Proof. (i) Let $l_{0} F_{[n+1]}(x)$ and $u_{0} F_{[n+1]}(x)$ be finite. Then there exist two real numbers $m$ and $M$ such that

$$
m<l_{0} F_{[n+1]}(x) \leqslant u_{0} F_{[n+1]}(x)<M .
$$

Hence when $h \neq 0$ is sufficiently small in absolute value, one has

$$
m<\left[F(x+h)-\sum_{i=0}^{n} F_{[i]}(x) h^{i} / i !\right](n+1) ! / h^{n+1}<M,
$$

and hence for such $h$ one sees that the number

$$
\left[F(x+h)-\sum_{i=0}^{n} F_{[i]}(x) h^{i} / i !\right] n ! / h^{n}
$$


lies between $m h /(n+1)$ and $M h /(n+1)$, both of which tends to zero as $h \rightarrow 0$. Hence the expression (4) tends to zero as $h \rightarrow 0$. From this fact one sees easily that

$$
F_{(i)}(x)=F_{[i]}(x) \text { for } i=0,1,2, \ldots, n,
$$

completing the proof.

(ii) By the definition of $F_{[n]}(x)$, we see that there exists a nonnegative integer $k_{0}$ such that, on a neighborhood of $x$, the function $F^{\left(-k_{0}\right)}$ has $\left(k_{0}+n\right)$ th Peano derivative at $x$, i. e. $\left(F^{\left(-k_{0}\right)}\right)_{\left(k_{0}+n\right)}(x)=F_{[n]}(x)$, and then by Lemma 1 , we see that whenever $k \geqslant k_{0}$ we have

$$
\left(F^{(-k)}\right)_{(k+n)}(x)=F_{[n]}(x),
$$

so that $l\left(F^{(-k)}\right)_{(k+n+1)}(x)$ makes sense since $F_{[n]}(x)$ is finite. If $k=0$, then inequalities hold trivially and the proof is done. If $k>0$, then note that

$$
\left(F^{(-k)}\right)_{(k+n-i)}(x)=F_{[n-i]}(x) \text { for } 0 \leqslant i \leqslant n,
$$

and furthermore, for $j=0,1,2, \ldots, k$, we have $\left(F^{(-k)}\right)^{(j)}=F^{(-k+j)}$ on a neighborhood of $x$ since $F$ is continuous on such a neighborhood by the definition of $F_{[n]}(x)$ and the convention of the notation $F^{(-k)}$. Then using the argument in the proof of Lemma $1, k$ times, one shows that the inequalities (3) hold, and the proof is completed.

From the proof, we see that (i) in Lemma 2 holds true even for $n=0$, and (ii) there also holds true for $n=0$ provided that the function $F$ is assumed to be continuous (0th differentiable) not only at $x$ but also on a neighborhood of $x$ (so that $\left(F^{-k}\right)$ makes sense). Hence from Definition 1 and Lemma 2 we have the following result:

LEMMA 3. If $F_{[n]}(x)$ exists, then $l_{0} F_{[n]}(x) \leqslant F_{[n]}(x) \leqslant u_{0} F_{[n]}(x)$.

Note that the inequalities in Lemma 3 may be restrictive. For example, let $F(x)=3 x^{2} \sin (1 / x)-x \cos (1 / x)$ when $x \neq 0$ and let $F(0)=0$; then $F_{[1]}(0)=0$, while $l_{0} F_{[1]}(0)=-1<0<1=u_{0} F_{[1]}(0)$ (cf. Remark 2).

Now, we come to consider some properties for the generalized Peano derivatives on intervals. First, we show that Theorems $\mathrm{A}_{n}, \mathrm{~B}_{n}$ and $\mathrm{C}_{n}$ can be extended to the generalized Peano derivative.

THEOREM 1. If a function $F$ is generalized nth Peano differentiable at every point of an interval, then, on the interval, $F_{[n]}$ is in the first class of Baire.

Proof. (This has been given in [9]. It seems worthwhile to reproduce it here to show some of the characteristics involved and also for the sake of completeness.) Let $x$ be a fixed arbitrary point in the interval concerned. Then there exists a nonnegative integer $k$, depending on $x$, such that $G \equiv F^{(-k)}$ has the $(k+n)$ th Peano derivative at $x$ which is equal to $F_{[n]}(x)$; i.e.

where

$$
F_{[n]}(x)=\lim _{h \rightarrow 0} Q(x, h)(k+n) ! / h^{k+n},
$$

$$
Q(x, h)=G(x+h)-\sum_{i=0}^{k+n-1} G_{(i)}(x) h^{i} / i !
$$


Then, it follows that

$$
\lim _{a \rightarrow 0} Q(x, a u) / a^{k+n}=F_{[n]}(x) u^{k+n} /(k+n) !
$$

uniformly for $u$ in any bounded set.

Now let us take $\varphi$ to be an infinitely differentiable function with compact support and such that $\int_{-\infty}^{\infty} \varphi(t) d t=1$. It then follows from (5) that we have

$$
\begin{aligned}
\lim _{a \rightarrow 0} \int_{-\infty}^{\infty} Q(x, a u) \varphi^{(k+n)}(u) d u / a^{k+n} \\
=F_{[n]}(x) \int_{-\infty}^{\infty} u^{k+n} \varphi^{(k+n)}(u) d u /(k+n) ! .
\end{aligned}
$$

Note that using integration by parts $i$ times, one has

$$
\int_{-\infty}^{\infty} u^{i} \varphi^{(k+n)}(u) d u= \begin{cases}0 & \text { when } 0 \leqslant i<k+n, \\ (-1)^{k+n}(k+n) ! & \text { when } i=k+n,\end{cases}
$$

by the facts that $\varphi$ has compact support and $\int \varphi(t) d t=1$. Hence (6) reduces to

$$
\lim _{a \rightarrow 0} \int_{-\infty}^{\infty} G(x+a u) \varphi^{(k+n)}(u) d u / a^{k+n}=(-1)^{k+n} F_{[n]}(x) .
$$

Again, using integration by parts $k$ times, the above equality becomes

$$
\lim _{a \rightarrow 0}(-1)^{k} \int_{-\infty}^{\infty} F(x+a u) \varphi^{(n)}(u) d u / a^{n}=(-1)^{k+n} F_{[n]}(x),
$$

or

$$
F_{[n]}(x)=\lim _{a \rightarrow 0} H_{a}(x)
$$

where

$$
H_{a}(x)=\int_{-\infty}^{\infty} F(x+a u) \varphi^{(n)}(u) d u /(-a)^{n} .
$$

Note that $H_{a}(x)$ is independent of $k$, and is continuous in $x$ for each $a \neq 0$. Hence equation (7) shows that $F_{[n]}$ is in the first class of Baire.

To generalize Theorems $\mathrm{B}_{n}$ and $\mathrm{C}_{n}$, we prove the following result first:

THEOREM 2. Let $F$ be a function continuous on the interval I. If $F_{[1]}(x)$ exists and is greater than zero for nearly every $x$ in $I$, then $F$ is strictly increasing on $I$.

Proof. Let $G=F^{(-1)}$ on $I$. Then $G^{\prime}=G_{(1)}=F$ on $I$. If $F_{[1]}(x)=F_{(1)}(x)=F^{\prime}(x)$, then by Lemma 1 one has $u G_{(2)}(x)=G_{(2)}(x)=F^{\prime}(x)$; if $F_{[1]}(x)$ exists but $F^{\prime}(x)$ does not exist, then there exists a positive integer $k$ such that $F_{(k+1)}^{(-k)}(x)=F_{[1]}(x)$ so that $G_{(2)}(x)=F_{[1]}(x)$ when $k=1$, and when $k>1$ one has $u G_{(2)}(x) \geqslant F_{(k+1)}^{(-k)}(x)$ $\left(=F_{[1]}(x)\right)$ by applying Lemma $1(k-1)$ times. This shows that $u G_{(2)}(x) \geqslant F_{[1]}(x)$ $>0$ for nearly every $x$ in $I$. Then by Lemma 1 in Verblunsky's paper [21], one concludes that $G$ is convex on $I$, and hence $G^{\prime}=F$ is monotone nondecreasing on $I$. Then $F$ must be strictly increasing on $I$, for otherwise $F$ would be constant on a nondegenerated interval and then $F^{\prime}(x)$ would be zero there, which would contradict the "greater than zero" condition. 
THEOREM $2^{*}$. Let $F$ be continuous on the interval I. If $F_{[1]}(x)$ exists and $\neq-\infty$ for nearly every $x$ in $I$ and $F_{[1]}(x) \geqslant 0$ for almost every $x$ in $I$, then $F$ is monotone nondecreasing on $I$.

Proof. This follows from Theorem 2 by a standard argument (cf. [10 and 15], where Verblunsky's Theorem 1 in [21] was used to prove Theorems $\mathrm{B}_{n}$ and $\mathrm{C}_{n}$ (in the last section) for approximate Peano derivatives) and hence is omitted here.

Now, generalizing both Theorems $\mathrm{B}_{n}$ and $\mathrm{C}_{n}$ in the last section, we have the following result:

THEOREM 3. Suppose that $F_{[n]}$ exists and is finite on the $I=[a, b]$. on $I$.

$\left(\mathrm{i}_{n}\right)$ If $F_{[n]}$ is bounded on one side in $I$, then $F^{(n)}$ exists (and, of course, equals $F_{[n]}$ )

(ii $\left.{ }_{n}\right)$ If $u_{0} F_{[n+1]}(x) \geqslant 0$ for almost every $x$ in I and $u_{0} F_{[n+1]}(x)>-\infty$ for nearly every $x$ in $I$, then $F_{[n]}$ is monotone nondecreasing and continuous on $I$.

To prove this, let us denote Theorem 2 as $\left(\mathrm{ii}_{0}\right)$. Then we will prove $\left(\mathrm{i}_{n}\right)$ by using (ii ${ }_{k}$ ) for $0 \leqslant k<n$, and also using $\left(\mathrm{i}_{n-1}\right)$ when $n \geqslant 2$; and then we will prove $\left(\mathrm{ii}_{n}\right)$ by using $\left(i_{n}\right)$.

Proof of $\left(i_{n}\right) . F_{[n]}$, being bounded on one side in $I$, we may assume, without loss of generality, that $F_{[n]}>0$ on $I$. Then by $\left(\mathrm{ii}_{n-1}\right), F_{(n-1)}$ is increasing on $I$. When $n=1, F_{[n-1]}=F_{[0]}$ is just the continuous function $F$. When $n \geqslant 2, F_{[n-1]}$ is bounded on $I$ and hence by $\left(\mathrm{i}_{n-1}\right)$, one has $F^{(n-1)}=F_{[n-1]}$ on $I$. We will show that the ordinary $F_{(n)}$ exists and equals $F_{[n]}$ on $I$. Then it follows from Theorem $\mathrm{B}_{n}$ that $F^{(n)}=F_{(n)}\left(=F_{[n]}\right)$ on $I$, and the proof is then completed.

Let us start to prove that $F_{(n)}(a)=F_{[n]}(a)$. To this end, note that by adding a suitable polynomial of degree less than $n$ without changing the existences and the values (if they exist) of both $F_{[n]}$ and $F_{(n)}$, we may assume $F_{(l)}(a)=F^{(l)}(a)=$ $F_{[l]}(a)=0$ for $l=0,1,2, \ldots, n-1$. Now, let $k$ be the smallest nonnegative integer such that $\left(F^{-k}\right)_{(k+n)}(a)=F_{[n]}(a)$. We will show that $k=0$, so that $F_{[n]}(a)$ is just the ordinary $F_{(n)}(a)$. Suppose, to the contrary, that $k>0$. Let us take $F^{(-k)} \equiv f$ to be the $k$ th fold Riemann integral of $F$ with $f^{(l)}(a)=0$ for $l=0,1,2, \ldots, k-1$. Note that by Lemma 1 (in §2) we have $f_{(k+l)}(a)=F_{(l)}(a)\left(=F_{[l]}(a)\right)$ for $l=0,1,2, \ldots$, $n-1$ since $F_{(n-1)}(a)$ exists. Thus, we have $f_{(m)}(a)=0$ for $m=0,1,2, \ldots, k+n-1$; and also note that we have $f_{(k+n)}(a)\left(=F_{[n]}(a)\right)>0$. Furthermore, since $F_{(n-1)}$ $\left(=F^{(n-1)}\right)$ exists on $I$, we also have, by Lemma 1 again, that $f_{(k+n-1)}=F_{(n-1)}$ on a neighborhood of $a$, and hence $f_{(k+n-1)}$ is increasing there. Then by applying Theorem $\mathrm{C}_{l}$ for $l=k+n-2, k+n-3, \ldots, 3,2,1$, one has that $f_{(l)}$ is increasing on a neighborhood of $a$ for $l=k+n-2, k+n-3, \ldots, 3,2,1$. In particular, $f_{(1)}$ is increasing there. Then, by Lemma 2 in Verblunsky's paper [21,], one concludes that $\left(f_{(1)}\right)_{(k+n-1)}(a)$ exists and equals $f_{(k+n)}(a)=F_{[n]}(a)$. Then we have

$$
\left(F^{(-k+1)}\right)_{(k-1+n)}(a)=\left(f_{(1)}\right)_{(k+n-1)}(a)=F_{[n]}(a),
$$

contradicting the definition of $k$.

Thus, we have proved that $F_{(n)}(a)=F_{[n]}(a)$. Since the point $a$ in the above argument can be replaced by any point in $[a, b]$, the proof of $\left(\mathrm{i}_{n}\right)$ is hence completed. 
Proof of $\left(\right.$ ii $\left._{n}\right)$. Let $G$ denote the set of all points $x$ in $[a, b]$ such that there exists a neighborhood $I_{x}$ of $x$ such that $F_{[n]}$ is monotone nondecreasing and continuous on $I_{x} \cap[a, b]$. Then (relative to $I=[a, b]$ ) $G$ is open and hence $I \sim G$ is closed. If we prove that $I \sim G$ is empty, then by a compactness argument, we see that $F_{[n]}$ is monotone nondecreasing and continuous on $I$. Suppose to the contrary that $E \equiv I$ $\sim G$ is not empty. Since $F_{[n]}$ is in the first class of Baire (by Theorem 1), there exists a point $x$ in $E$ such that $F_{[n]}$ is continuous at $x$ relative to the set $E$. Therefore, there exists an open interval $I_{0}$ containing $x$ such that $F_{[n]}$ is bounded on $P \equiv \operatorname{cl} I_{0} \cap E$, where cl $I_{0}$ denotes the closure of $I_{0}$. We will show that $F_{[n]}$ is monotone nondecreasing and continuous in $\mathrm{cl} I_{0} \cap I$ (and hence in $I_{0} \cap I$ ), so that $x \in G$, a contradiction. The proof is then completed.

Denote $J=\operatorname{cl} I_{0} \cap I$. Then $J$ is a compact and nondegenerated interval containing the closed set $P$. On each closed interval contained in $J \sim P$, the function $F_{[n]}$ is easily seen to be monotone nondecreasing and continuous. Thus, $F_{(n)}$ is monotone nondecreasing and continuous on each open interval contiguous to $P$. Let $(c, d)$ be such an open interval. Then for any $\alpha$ in $(c, d)$, one has that $F_{(n)}$ is bounded from above on $[c, \alpha]$ and from below on $[\alpha, d]$. Hence, by $\left(\mathrm{i}_{n}\right), F_{[n]}$ is just the ordinary $F^{(n)}$ on both $[c, \alpha]$ and $[\alpha, d]$. The ordinary derivative $F^{(n)}$, being monotone on the open interval $(c, d)$, must then be so on the closed interval $[c, d]$ and continuous there since the ordinary derivative has the Darboux property. Thus, we have shown that $F_{[n]}$ is continuous on the closure of each interval contiguous to $P$. Then, $F_{[n]}$, being bounded on $P$, must be bounded on the whole interval $J$. Then, by ( $\left.i_{n}\right)$ again, $F_{[n]}$ is just the ordinary $F^{(n)}$ on $J$. Then $u F_{(n+1)}$ makes sense and is just $u_{0} F_{[n+1]}$ on $J$. Therefore, we have $u F_{(n+1)} \geqslant 0$ almost everywhere on $J$ and $u F_{(n+1)}>$ $-\infty$ nearly everywhere on $J$. So that by Theorem $\mathrm{C}_{n}$, we conclude that $F_{[n]}$ $\left(=F_{(n)}=F^{(n)}\right)$ is monotone nondecreasing and continuous on $J$. This completes the proof.

Many interesting properties for the generalized Peano derivatives can be obtained by using Theorems 1 to 3 in a rather routine manner. For example, we have the following:

THEOREM 4. Let $F_{[n]}$ exist and be finite on the interval $[a, b]$. Then $F_{[n]}$ has both the Darboux property and the Denjoy property on $[a, b]$.

THEOREM 5. If $F_{[n]}=0$ on the interval, then $F$ is a polynomial of degree less than $n$.

Proof of Theorem 4. Note that $F_{[n]}$ is in the first class of Baire by Theorem 1 . By a characterization of Baire one Darboux functions due to Neugebauer (cf. [1]), in order to show that $F_{[n]}$ has the Darboux property, we need only show that the components of the sets $E^{\Lambda}=\left\{x: F_{[k]}(x) \geqslant \Lambda\right\}$ and $E_{\Lambda}=\left\{x: F_{[n]}(x) \leqslant \Lambda\right\}$ are closed for each real number $\Lambda$. So suppose $F_{[n]} \geqslant \Lambda$ on the open interval $(c, d)$. We want to show $F_{[n]}(c) \geqslant \Lambda$ and $F_{[n]}(d) \geqslant \Lambda$. Since $F_{[n]}$ is bounded below on $(c, d)$ it is bounded below on $[c, d]$. Thus by Theorem $3\left(\mathrm{i}_{n}\right), F^{(n)}=F_{[n]}$ on $[c, d]$. But $F^{(n)}$ has the Darboux property so that $F^{(n)}(c) \geqslant \Lambda$ and $F^{(n)}(d) \geqslant \Lambda$. Hence $F_{[n]}(c)$ $\left(=F^{(n)}(c)\right) \geqslant \Lambda$ and $F_{[n]}(d)\left(=F^{(n)}(d)\right) \geqslant \Lambda$. Thus, the components of $E^{\Lambda}$ are closed. Similarly, the components of $E_{\Lambda}$ are closed. Hence, $F_{[n]}$ has the Darboux property. 
To show that $F_{[n]}$ has the Denjoy property on $[a, b]$ (i.e. the property $A$ is [23]), it suffices, by a theorem in [23], to show that for each subinterval $J$ (of $[a, b]$ ) on which $F_{[n]}$ is bounded on one side, $F_{[n]}$ has the Denjoy property on $J$. But by Theorem $3\left(\mathrm{i}_{n}\right), F_{[n]}$ is just the ordinary $F^{(n)}$ on such subintervals and hence has the Denjoy property there. This completes the proof.

Proof of Theorem 5. By Theorem $3\left(\mathrm{i}_{n}\right), F_{[n]}$ is just the ordinary $F^{(n)}$ on the interval. Thus, $F^{(n)}=0$ on the interval, and hence $F$ is a polynomial of degree less than $n$.

We end this section by proving the following result, which will be fundamental to our discussion in the next section:

THEOREM 6. Let $m$ and $n$ be nonnegative integers with $m \leqslant n$, and let $F$ and $G$ be functions such that $F$ is generalized nth Peano differentiable and $G$ is generalized mth Peano differentiable and $F_{[n]}=G_{[m]}$ on the interval $I=[a, b]$. Then

(i) there exists a polynomial $p$ of degree less than $m$ such that

$$
G=F_{[n-m]}+p \text { on } I
$$

(ii) if $k$ is a positive integer and $G_{[m+k]}(x)$ exists for some $x \in I$, then $F_{[n+k]}(x)$ exists and equals $G_{[m+k]}(x)$.

Proof. Let $g$ be any $(n-m)$-fold indefinite Riemann integral of $G$ on $I$ (if $m=n$, this just means $g=G)$, so that we have $g^{(n-m)}=G$ on $I$. Furthermore, $g_{[n]}=G_{[m]}=F_{[n]}$ on $I$. Then by the finiteness of $F_{[n]}$ and $G_{[m]}$, we have $(g-F)_{[n]}$ $=0$ on $I$. Hence $g-F$ on $I$ is a polynomial of degree less than $n$ by Theorem 5 . Thus, $p \equiv(g-F)^{(n-m)}$ is a polynomial of degree less than $m$. Then, one has $G=g^{(n-m)}=F_{[n-m]}+p$ on $I$, proving (i). The part (ii) follows easily from (i) since $p_{[m+k]}=p^{(m+k)}=0$ on $I$. [Note that here by a polynomial of degree less than zero, we mean the constant function 0 .]

4. Absolute generalized Peano derivatives. Using finite Peano derivatives, $\mathbf{M}$. Laczkovich has recently studied an interesting concept, called the absolute Peano derivative [8]. Following his step, but using the generalized Peano derivatives instead of the ordinary ones, we have the absolute generalized Peano derivative. (See Definition 2 below.) We show that every absolute generalized Peano derivative on a compact interval is just a generalized Peano derivative (corollary to Theorem 7). In particular, Laczkovich's absolute Peano derivatives are generalized Peano derivatives, and hence have those properties studied in the previous section.

Before giving the definition, we would like to make a minor point clear. In [8], a function $f$ is said to have, at the point $x$, an absolute Peano derivative equal to a real number $A$ (in symbols, $f^{*}(x)=A$ ) if there exist a nonnegative integer $n$ and a function $g$ such that (i) $g_{(n)}=f$ on a neighborhood of $x$, and (ii) $g_{(n+1)}(x)=A$. Note that in the context of [8], when $n=0$, the condition (i) (i.e. $g_{(0)}=f$ on a neighborhood of $x$ ) means only that $g=f$ there without any continuity condition for the function $f$ on the whole neighborhood. On the other hand, by our convention here, when we write $g_{[0]}=f$ on a neighborhood, we mean not only that $g=f$ there but also $g\left(=g_{[0]}=f\right)$ is continuous there. Thus, in the following definition we have 
singled out the case when $f$ is differentiable only at $x$ without any other continuity condition on a whole neighborhood. With this, we are assured that every absolute Peano derivative is an absolute generalized Peano derivative.

DEFINITION 2. Let $f$ be a (real-valued) function defined in a neighborhood of $x$. We say that $f$ is absolutely generalized Peano differentiable at $x$ if either $(\alpha) f$ is differentiable at $x$, or $(\beta)$ there exist a nonnegative integer $n$ and a function $g$ such that (i) $g_{[n]}=f$ on a neighborhood of $x$ and, (ii) $g$ is generalized $(n+1)$ th Peano differentiable at $x$.

REMARK 3. Suppose that a function $f$ is differentiable at $x$ and there exist a nonnegative integer $n$ and a function $g$ such that $g_{[n]}=f$ on a neighborhood of $x$. Then $g_{[n+1]}(x)$ exists and equals $f^{\prime}(x)$.

Proof. If $n=0$, then $g_{[0]}=f$ on a neighborhood of $x$, and hence by our convention, $f$ is continuous there, so that $f^{\prime}(x)$ exists means $g_{[1]}(x)=f_{[1]}(x)=f^{\prime}(x)$ according to Definition 1 in the previous section. Thus, we are done when $n=0$. Suppose that $n \geqslant 1$. Note that $f$ is differentiable at $x$ implies that $f$ is continuous at $x$ and hence $f$ is bounded in a neighborhood of $x$. Hence $g_{[n]}(=f)$ is bounded on a neighborhood of $x$, too. Then by Theorem 3, we have $g^{(n)}=g_{[n]}=f$ on a neighborhood of $x$. Therefore $g$ is continuous on that neighborhood, and $f^{\prime}(x)=g^{(n+1)}=$ $g_{[n+1]}(x)$, completing the proof.

It follows from Remark 3 and Theorem 6 (in the previous section) that if a real-valued function $f$ is absolutely generalized Peano differentiable at $x$, then there exists a unique real number $A$ such that either $(\alpha) F^{\prime}(x)=A$, or $(\beta)$ there exist a nonnegative integer $n$ and a function $g$ such that (i) $g_{[n]}=f$ on a neighborhood of $x$, and (ii) $g_{[n+1]}(x)=A$. This unique number $A$ is called the absolute generalized Peano derivative of $x$, and is denoted as $f^{[*]}(x)$.

THEOREM 7. Let $f$ be a function such that $f^{[*]}$ exists on a compact interval $[a, b]$. Then there exist a positive integer $n$ and a function $g$ such that $g_{[n]}=f$ on $[a, b]$.

The proof of Theorem 7 to be given below is a standard compactness argument based on the following lemma, of which the idea is inspired by the proof of Theorem 3 in [8].

LEMMA 4. Let $f^{[*]}$ exist on a neighborhood of $x$. Then there exist a positive integer $n$ and a function $F$ such that $F_{[n]}=f$ on some neighborhood of $x$.

Proof. As $f^{[*]}(x)$ exists, either $(\alpha) f^{\prime}(x)=f^{[*]}(x)$, or $(\beta)$ there exist a nonnegative integer $n$ and a function $g$ such that (i) $g_{[n]}=f$ on a neighborhood of $x$, and (ii) $g_{[n+1]}(x)=f^{[*]}(x)$. If it is the case $(\beta)$, we are done by taking $F=g$ when $n \geqslant 1$, and $F=g_{[0]}^{(-1)}$ when $n=0$. Therefore, we assume that it is the case $(\alpha)$. Then, $f$, being differentiable at $x$, is bounded on a neighborhood of $x$, say $I_{x}$. We can and do take $I_{x}$ so small an interval that it is contained in the neighborhood of $x$ on which the existence of $f^{[*]}$ is assumed. We will complete the proof by showing that $f$ is a derivative on $I_{x}$. To this end, note that since $f^{[*]}$ exists on $I_{x}$, for each $t \in I_{x}$, either ( $\alpha) f(t)=f^{[*]}(t)$, or $(\beta)$ there exist a nonnegative integer $n$ and a function $g$ such that $g_{[n]}=f$ on a neighborhood of $t$ and $g_{[n+1]}(t)=f^{[*]}(t)$. If it is $(\beta)$ and $n=0$, 
then $\left[g^{(-1)}\right]^{\prime}=f$ on a neighborhood of $t$ since $f=g_{[0]}$ on such a neighborhood means $f$ is continuous there. If it is $(\beta)$ and $n \geqslant 1$, then, since $f$ is bounded on $I_{x}$, it follows from Theorem 3, that $g^{(n)}=g_{[n]}=f$ on a neighborhood of $t$. Thus, $f$ is a derivative on a neighborhood of $t$ when it is $(\beta)$. Then, denoting $O=\left\{t \in I_{x}: f\right.$ is a derivative on a neighborhood of $t\}$ and $C=I_{x} \sim O$, we see that for each $t \in C$, we must have $f^{\prime}(t)=f^{[*]}(t)$, and, in particular, $f$ is continuous at each $t \in C$. From this fact, one sees that on $I_{x}$, the function $f$ is in the first class of Baire. In fact, let $P$ be any nonempty set closed in $I_{x}$; if $P$ contains a point in $C$, then $f \mid P$ is continuous at that point; if $P$ does not contain any points of $C$, then $P$ is a subset of $O$ which is easily seen to be open in $I_{x}$, and hence $f \mid P$ is continuous at some point of $P$ since a derivative is in the first class of Baire; thus every nonempty closed subset $P \subset I_{x}$ contains a point at which $f \mid P$ is continuous, and hence on $I_{x}, f$ is in the first class of Baire. Now, $f$, being both bounded and in the first class of Baire on $I_{x}$, is summable on each bounded subinterval of $I_{x}$. Letting $F(t)=\int_{x}^{t} f(u) d u$ for $t \in I_{x}$, we see that $F^{\prime}(t)=f(t)$ for each $t \in I_{x}$. In fact, if $t \in C$, then $F^{\prime}(t)=f(t)$ simply because $f$ is continuous at $t$; if $t \in O$ then there exists a function $G$ such that $G^{\prime}=f$ on a neighborhood of $t$, and then on this neighborhood one sees easily that $G$ and $F$ can be different by at most a constant term so that, in particular, one has $F^{\prime}(t)=G^{\prime}(t)$ $=f(t)$. Thus, we have showed that $f$ is a derivative on $I_{x}$, completing the proof.

Proof of Theorem 7. Let $H=\{u: u \in(a, b]$ and there exist a positive integer $n$ and a function $g$ such that $g_{[n]}=f$ on $\left.[a, u]\right\}$. Applying Lemma 4 to a neighborhood of $a$, we see that $H$ is nonempty. Thus, denoting $c=\sup H$, we have $a<c \leqslant b$. Applying Lemma 4 again to a neighborhood of $c$, one has a positive integer $k$ and a function $F$ such that $F_{[k]}=f$ on a neighborhood of $c$, say on $[c-\delta, c+\delta] \cap[a, b]$ for some $\delta>0$. Since $c>a$, we have a point $x \in H$ such that $x \in(c-\delta, c)$. Then there exist a positive integer $n$ and a function $G$ such that $G_{[n]}=f$ on $[a, x]$. Denoting $m=\max \{k, n\}$, we have

$$
\left(F^{(-m+k)}\right)_{[m]}=\left(G^{(-m+n)}\right)_{[m]} \text { on }[c-\delta, x] .
$$

Hence by Theorem 5 , there exists a polynomial $p$ of degree less than $m$ such that $F^{(-m+k)}=G^{(-m+n)}+p$ on $[c-\delta, x]$. Then, letting $g$ be the function defined by

$$
g= \begin{cases}G^{(-m+n)} & \text { on }[a, x], \\ F^{(-m+k)}-p & \text { on }[x, \min \{c+\delta, b\}],\end{cases}
$$

we have $g_{[m]}=f$ on $[a, \min \{c+\delta, b\}]$. Hence $\min \{c+\delta, b\} \in H$. Then by the definition of $c$, we have $b=c$, and the proof is completed.

COROllary. Every absolute generalized Peano derivative on a compact interval is a generalized Peano derivative there. In particular, every Laczkovich's absolute Peano derivative on a compact interval is a generalized Peano derivative.

Proof. Let $f^{[*]}$ exist on the compact interval $[a, b]$. Then by Theorem 7 , there exist a positive integer $n$ and a function $g$ such that $g_{[n]}=f$ on $[a, b]$. It then follows from Definitions 1, 2, Remark 3 and Theorem 6 that $g_{[n+1]}=f^{[*]}$ on $[a, b]$. 
Remark 4. Laczkovich [8] has constructed an absolute Peano derivative on an interval which is not an ordinary Peano derivative of any order. Thus, it follows from the above corollary that the generalized Peano derivative on an interval is a genuine generalization of the ordinary Peano derivative of any order. We remark, in passing, that it can be seen easily that a function is a Laczkovich's absolute Peano derivative on an interval if and only if it is pointwisely Cesàro continuous there as defined in [9], where the concept arises naturally in a consideration of Burkill's scale of the Cesàro-Perron integrals [5]. The author would like to take the opportunity to thank Professor P. S. Bullen for directing his attention to Laczkovich's paper [8].

5. For further investigations. It is know (cf. [ 2 or 11]) that directly using Peano derivatives and derivates, one can obtain a scale of consistent integrals, which is equivalent to Burkill's scale of the Cesàro-Perron integrals [5]. Here, we briefly describe how a scale of consistent generalized integrals can be obtained by using the generalized Peano derivatives and "derivates". We begin with the definition of a suitable generalized Peano derivate.

Let $F$ be generalized $(n-1)$ th Peano differentiable at $x$. Then the upper generalized $n$th Peano derivate of $F$ at $x$, denoted as $u F_{[n]}(x)$, is defined as follows: $u F_{[n]}(x)=u F_{(1)}(x)$ when $n=1, u F_{[n]}(x)=u\left(F^{(-k)}\right)_{(k+n)}(x)$ when $n>1$, where $k$ is the smallest nonnegative integer such that $\left(F^{(-k)}\right)_{(k+n-1)}(x)=F_{[n-1]}(x)$. The lower generalized $n$th Peano derivate of $F$ at $x$, denoted as $l F_{[n]}(x)$, is defined in a similar manner. Then, when $F$ is generalized $(n-1)$ th differentiable at $x$ for $n>1$, one has (cf. Lemma 2)

$$
\begin{aligned}
l_{0} F_{[n]}(x) & \leqslant l F_{[n]}(x) \leqslant l\left(F^{(-k)}\right)_{(k+n)}(x) \\
& \leqslant u\left(F^{(-k)}\right)_{(k+n)}(x) \leqslant u F_{[n]}(x) \leqslant u_{0} F_{[n]}(x)
\end{aligned}
$$

whenever $k$ is sufficiently large. Note that the above inequalities hold true when $n=1$ provided that $F$ is known to be continuous not only at $x$ but also on a neighborhood of $x$.

Let $f$ be an extended real-valued function defined on a compact interval $[a, b]$ and is finite almost everywhere there. A finite function $M$ is said to be a $G P_{n-1}$-major function of $f$ on $[a, b]$ if there exists a continuous function $F$ such that the following hold:

(i) $F_{[n-1]}=M$ on $[a, b]$,

(ii) $M(a)=0$,

(iii) $l F_{[n]} \geqslant f$ almost everywhere on $[a, b]$,

(iv) $l F_{[n]}>-\infty$ nearly everywhere on $[a, b]$.

If $-m$ is a $G P_{n-1}$-major of $-f$ on $[a, b]$, we say that $m$ is a $G P_{n-1}$-minor of $f$ on $[a, b]$. Then, using Theorem 3(ii), and following the usual process of defining an integral of Perron type, one can define a $G P_{n-1}$-integral. Thus, we have a scale of integrals, i.e. $G P_{n-1}$-integral for $n=1,2,3, \ldots$ Note that the $G P_{0}$-integral is just the classical Perron integral, while the $G P_{n}$-integral is more general than Burkill's $C_{n} P$-integral. Note also that, similar to Burkill's scale, the new scale of integrals is consistent, i.e. if a function $f$ is $G P_{n-1}$-integrable on $[a, b]$, then it is also $G P_{n}$-integrable there, and in this case these two integrals are equal. 
For Burkill's $C_{n} P$-integral, Sargent has given some interesting equivalent definitions of Denjoy type in [19] (cf. [22]) and in [20]. We expect that such characterizations can be somehow extended to the more general $G P_{n}$-integral. We also expect that such characterizations for the $G P_{n}$-integral may be useful in knowing whether the generalized Peano derivative has the Zahorski property, the property $Z$, or the oscillatory behavior mentioned in the introduction. (Cf. Added in proof.)

The development of the generalized Peano derivative is based on Lemma 1, which fails to hold true (cf. Remark 1) when the Peano derivatives and derivates are replaced by the approximate ones. However, subject to a finiteness condition (see $(*)$ below), we can improve the inequalities in Lemma 1 a little bit. In fact, one can prove the following:

LEMMA 5. Let $f$ and $g$ be real-valued functions such that $g_{(1), \text { ap }}=f$ on a neighborhood of $x$. Suppose that $u f_{(n), \text { ap }}(x)$ makes sense for a positive integer $n$. Then $u g_{(n+1) \text {,ap }}(x)$ makes sense and

$$
l f_{(n), \mathrm{ap}}(x) \leqslant l_{0} g_{(n+1), \mathrm{ap}} \leqslant u_{0} g_{(n+1), \text { ap }}(x) \leqslant u f_{(n), \mathrm{ap}}(x)
$$

provided that the following condition holds:

$$
\text { both } u_{0} f_{(k) \text {,ap }}(x) \text { and } l_{0} f_{(k) \text {,ap }}(x) \text { are finite for } 1 \leqslant k \leqslant n \text {. }
$$

Here, the notation $u F_{(n) \text {,ap }}(x)$ denotes the upper approximate $n$th Peano derivate of $F$ at $x$ (cf. Remark 1); and the notation $u_{0} F_{(n) \text {,ap }}(x)$ denotes the number obtained by replacing the "limsup ap" in the definition of $u F_{(n) \text {,ap }}(x)$ by the ordinary "lim sup"; and the meaning of each of the $l F_{(n), \text { ap }}(x)$ and $l_{0} F_{(n) \text {,ap }}(x)$ is similar in an obvious way.

The proof of Lemma 5 is not much harder than that of Lemma 1 provided that the finite condition (*) is suitably used (cf. the proof of Lemma 2(i)). We omit it here. Instead, we conclude this note by posing a question related to Lemmas 1 and 5.

First, note again that in Lemma 5 we cannot even have the inequalities (instead of (9)),

$$
l f_{(n), \mathrm{ap}}(x) \leqslant l g_{(n+1), \text { ap }}(x) \leqslant u g_{(n+1), \mathrm{ap}}(x) \leqslant u f_{(n), \text { ap }}(x),
$$

if the finiteness condition $(*)$ is omitted. However, the condition $(*)$ is too strong in the sense that under this condition not only (10) holds but even (9) holds. What kind of "weaker" condition can replace condition $(*)$ in Lemma 5 so that (10) holds true while (9) may fail to be true? If such a "weaker" condition can be obtained, then, with a little care, one may develop the concept of "(absolute) generalized" approximate Peano derivatives, which will be strictly more general than the (absolute) generalized Peano derivatives discussed in this note.

ADDED IN PROOF. The generalized Peano derivatives do have the Zahorski property, the property $Z$, and the oscillatory property. Proofs of the results will appear somewhere else.

\section{REFERENCES}

1. B. S. Babcock, On the properties of the approximate Peano derivatives, Trans. Amer. Math. Soc. 212 (1975), 279-294.

2. J. A. Bergin, A new characterization of Cesàro-Perron integrals using Peano derivatives, Trans. Amer. Math. Soc. 228 (1977), 287-305. 
3. P. S. Bullen and S. N. Mukhopadhyay, Peano derivatives and general integrals, Pacific J. Math. 47 (1973), 43-58.

4. On the Peano derivatives, Canad. J. Math. 15 (1973), 127-140.

5. J. C. Burkill, The Cesàro-Perron scale of integration, Proc. London Math. Soc. (2) 39 (1935), $541-552$.

6. A. Denjoy, Sur l'intégration des coefficients différentiels d'ordre supérieur, Fund. Math. 25 (1935), 273-326.

7. M. J. Evans, $L_{p}$ derivatives and approximate Peano derivatives, Trans. Amer. Math. Soc. 165 (1972), 381-388.

8. M. Laczkovich, On the absolute Peano derivatives, Ann. Univ. Sci. Budapest. Eötvös Sect. Math. 21 (1978), 83-97.

9. C. M. Lee, Generalizations of Cesàro continuous functions and integrals of Perron type, Trans. Amer. Math. Soc. 266 (1981), 461-481.

10. On the approximate Peano derivatives, J. London Math. Soc. 12 (1976), 475-478.

11. An approximate extension of Cesàro-Perron integrals, Bull. Inst. Acad. Sinica 4 (1976), $73-82$.

12. , Note on the oscillatory behavior of certain derivatives, Real Anal. Exchange 4 (1979),

$178-183$.

13. C. M. Lee and R. J. O'Malley, The second approximate derivative and the second approximate Peano derivative, Bull. Inst. Math. Acad. Sinica 3 (1975), 193-197.

14. S. Lojasiewicz, Sur la valeur et la limite d'une distribution en un point, Studia Math. 16 (1957), 1-36.

15. S. N. Mukhopadhyay, On the approximate Peano derivatives, Fund. Math. 88 (1975), 133-143.

16. H. W. Oliver, The exact Peano derivative, Trans. Amer. Math. Soc. 76 (1954), 444-456.

17. R. J. O'Malley and C. E. Weil, The oscillatory behavior of certain derivatives, Trans. Amer. Math. Soc. 234 (1977), 467-481.

18. S. Saks, Theory of the integral, Warsaw, 1937.

19. W. L. C. Sargent, A descriptive definition of Cesàro-Perron integrals, Proc. London Math. Soc. (2) 47 (1942), 212-247.

20. On generalized derivatives and Cesàro-Denjoy integrals, Proc. London Math. Soc. (2) 52 (1951), 365-376.

21. S. Verblunsky, On the Peano derivatives, Proc. London Math. Soc. (3) 22 (1971), 313-324.

22. __ On a descriptive definition of Cesàro-Perron integrals, J. London Math. Soc. (2) 3 (1971), $326-333$.

23. C. E. Weil, On properties of derivatives, Trans. Amer. Math. Soc. 114 (1965), 363-376.

24. __ A property for certain derivatives, Indiana Univ. Math. J. 23 (1973), 527-536.

Department of Mathematics, University of Wisconsin-Milwaukee, Milwaukee, Wisconsin 53201 